\title{
Inducing Amnesia for Unwanted Memories through Subliminal Reactivation
}

\author{
(D)Zijian Zhu ${ }^{* 1}$, DMichael C. Anderson², (DYingying Wang ${ }^{* 3}$ \\ ${ }^{1}$ School of Psychology, Shaanxi Normal University, Xi'an 710062, Shaanxi, China \\ ${ }^{2}$ MRC Cognition and Brain Sciences Unit, University of Cambridge, Cambridge, UK \\ ${ }^{3}$ Department of Psychology and Behavioral Sciences, Zhejiang University, Hangzhou 310028, Zhejiang, China
}

November 28, 2021. Preprint v1.1

$\mathrm{T}$ raumatic memories contribute to psychological conditions such as post-traumatic stress disorder and phobias. Treatment of these disorders may benefit from techniques that reduce the accessibility of unwanted memories and their impact on cognition and emotion. Procedures such as retrieval suppression, associative interference, and reconsolidation disruption, though effective in inducing forgetting, involve exposure to the traumatic event, which is aversive and carries risks to the patient. But is explicit awareness of traumatic content truly necessary for effective voluntary forgetting? Recently, intentionally suppressing (i.e., stopping) retrieval of a memory in response to a reminder has been shown to temporarily interrupt hippocampal function. Disrupting hippocampal function through retrieval suppression induces an amnesic shadow that impairs the encoding and stabilization of unrelated "innocent bystander" memories that are activated near in time to people's effort to suppress retrieval. Building on this mechanism, we successfully disrupted retention of unpleasant memories by subliminally reactivating them within this amnesic shadow window (on 88 participants across two experiments). Following the characteristics of retrieval suppression, the amnesic shadow disrupted memory for the subliminally reactivated events and induced forgetting that generalized across retrieval cues. Critically, whereas unconscious forgetting occurred on these affective "innocent bystander" memories, the amnesic shadow itself was induced by conscious suppression of unrelated and benign neutral memories, avoiding direct conscious re-exposure of unwelcome content. Combining the amnesic shadow with subliminal reactivation may offer a new approach to forgetting trauma that bypasses the unpleasantness in conscious exposure to unwanted memories.

\section{Introduction}

Recurrent intrusive memories and ruminations are key symptoms in a range of psychiatric conditions, including post-traumatic stress disorder (PTSD), acute stress disorder, and obsessive-compulsive disorder (Brewin et al.,
2010). Treatments on these symptoms often emphasize gradual re-exposure to the major stressors. For instance, a widely used therapy for PTSD, exposure therapy, involves gradually confronting cues related to the traumatic event; and a second, the eye movement desensitization and reprocessing (EMDR) treatment approach requires that patients hold a mental image of the traumatic event in mind while visually tracking a bilateral stimulus (Bradley et al., 2005). Actual or imaginal re-exposure, although effective in reducing symptoms, can be aversive to patients. Reponses to aversive content can lead participants to prematurely terminate therapy and also involve additional risks to patients (Loerinc et al., 2015; Zayfert et al., 2005). Here we ask whether it is possible to reduce the intrusiveness of an unwanted memory while avoiding any requirement for people to consciously reexperience it.

To address the foregoing problem, we propose that an unwanted memory may be forgotten by subliminally reactivating it during a time window when hippocampal processing is actively inhibited by voluntary retrieval suppression. This surprising possibility follows from the neural mechanisms underlying retrieval suppression (Anderson \& Hulbert, 2021; Hulbert et al., 2016) and unconscious memory processing (Degonda et al., 2005; Henke, 2010). Research on retrieval suppression has found that intentionally suppressing (i.e., stopping) memory retrieval given a reminder to an event downregulates hippocampal activity; in doing so, retrieval suppression globally disrupts hippocampal functions such as the encoding, retrieval and stabilization of memories. Disrupting hippocampal processes mimics organic amnesia, triggering both retrograde and anterograde memory deficits. This effect, known as the amnesic shadow (Hulbert et al., 2016; Zhu \& Wang, 2021), occurs in the temporal surround of each retrieval suppression attempt (extending at least 5-10s before and after suppression), creating a window during which "innocent bystander" memories can be disrupted. To be affected by the amnesic shadow, however, a memory's retention must rely on ongoing hippocampal processing that gets prevented by suppression (Hulbert et al., 2016; Zhu \& Wang, 2021). Critically, evidence suggests that hippocampal traces may be reactivated without awareness. Indeed, 
the hippocampus mediates rapid associative memory re- 59 trieval without requiring consciousness and can be acti- 6 vated even by subliminally presented cues (Degonda et al., 6 2005; Duss et al., 2014; Jensen et al., 2015). Together, 62 these findings imply a striking possibility: it should be possible to forget a hippocampally dependent memory by subliminally exposing reminders to it during the amnesic shadow induced by retrieval suppression on unrelated memories.

To test this hypothesis, we measured whether retrieval suppression affected the accessibility of unrelated memories that were subliminally cued during the amnesic shadow. To induce suppression, we adapted the Think/Nothink (TNT) paradigm (Anderson \& Green, 2001). In our TNT task, people performed trials in which they received a reminder of a previously studied verbal memory item and were cued either to retrieve the associated word (Think trials) or to suppress its retrieval (No-think trials). Repeated No-think practice has been found to induce forgetting on the suppressed memories, a phenomenon known as suppression-induced forgetting (Anderson \& Green, 2001). Importantly, in prior work, these same suppression trials also are known to induce forgetting on entirely unrelated events (hereinafter called Bystander events) encoded or reactivated close in time to No-think trials (Anderson \& Hulbert, 2021). Therefore, retrieval suppression does not merely disrupt the particular suppressed memories, but rather reflects a broadly targeted suppression of regional activity within the hippocampus (Hulbert et al., 2016). Here, we inserted subliminal reminders (simple visual objects) to previously encoded Bystander events (scenes) in between two No-Think trials (see Figure 1) to maximize the chances that hippocampal processes would be adversely affected during the reminder; because the previous and subsequent No-Think trials should disrupt hippocampal processes, the reminder should fall within the amnesic shadow. The Bystander reminders appeared subliminally with a sandwich masking procedure (Degonda et al., 2005; Henke et al., 2003). Unbeknownst to participants, target scenes associated with the Bystander objects would be tested after the TNT task.

We predicted that the amnesic shadow induced by retrieval suppression would trigger forgetting on these unrelated bystander scenes that we subliminally cued within the shadow period. In addition, if bystander forgetting reflects disruption of the reactivated scene memory itself, forgetting on the delayed test should not only arise when we test the scene with the reminder cue used for subliminal reactivation, but also with an independent cue that was not subliminally re-exposed during the Think/No-Think task. To increase the clinical relevance of our amnesic shadow measurement, the scenes encoded in our object-scene bystander pairs were always aversive. The object reminder cues were selected to resemble an incidental object embedded in its paired scene to simulate natural situations associated with involuntary trauma recall (Küpper et al., 2014). The scene targets contained complex affective content allowing us to measure not only whether the scene was recalled (Identification), but also the level of detail that was accessible (Gist). We predicted that subliminally presenting reminders to these Bystander scenes during the amnesic shadow would impair performance on both Identification and Gist measures of Bystander scene recall in the final testing phase (see Figure 1), despite participants having had no awareness of the masked reminders during the preceding Think/No-Think task.

\section{Results}

\section{Experiment 1}

In Experiment 1, we tested whether the amnesic shadow could disrupt a Bystander memory that was subliminally reactivated by reminder cues. We applied a masking procedure to all the Think and to half of the No-think Bystanders so that participants could not consciously perceive the content of Bystander cues (Fig. 1B). For comparison, we presented half of the No-think Bystanders supraliminally as in our previous study (Zhu \& Wang, 2021). We performed an offline consciousness check at the end of the experiment to verify that participants could not identify masked bystander cues (Fig. 1C).

\section{Suppression induced forgetting on TNT pairs.}

We first tested the standard suppression-induced forgetting (SIF) effect on the TNT pairs themselves, verifying our manipulation. Recall accuracy varied significantly across our four conditions (i.e., Think, No-think conscious, No-think unconscious, and Control) (Fig. 2A, F(3,117) = $\left.8.85, \mathrm{p}<.001, \eta_{\mathrm{p}}{ }^{2}=0.19\right)$. As expected, retrieval suppression induced significant memory impairment on No-Think items when compared with the Control condition (Nothink conscious vs. Control: $\mathrm{t}(39)=-3.11, \mathrm{p}=.003$, Cohen's $\mathrm{d}=0.49$; No-think unconscious vs. Control: $\mathrm{t}(39)=$ $-2.52, \mathrm{p}=.016$, Cohen's $\mathrm{d}=0.40$ ), confirming that our retrieval suppression manipulation succeeded. Whereas retrieval during Think trials numerically increased final test performance for Think items, the improvement was not significant (Think vs. Control: $\mathrm{t}(39)=1.50, \mathrm{p}=.141$, Cohen's $d=0.24)$.

\section{The amnesic shadow impairs consciously and unconsciously} reactivated bystander scenes.

Of key interest is whether subliminally exposing Bystander cues within the window of the amnesic shadow would lead to an amnesic shadow effect on the later recall test. Before testing this, it was critical to exclude participants who might have identified or recognized the Bystander cues, despite our subliminal procedure. For this, we turned to the consciousness checking phase at the end of the experiment during which participants were overtly directed to identify the masked objects and then make old/new judgement on them. On this task, if a participant could recognize $66.7 \%$ or greater (a one-tailed 5\% cut-off of $66.7 \%$ ) of masked objects they were considered likely to have perceived Bystander objects in the earlier TNT phase and so were excluded and replaced. Two participants were excluded and replaced with this procedure. On this ba- 
A

Learning phase

Think/No-think phase + bystanders

Testing phase
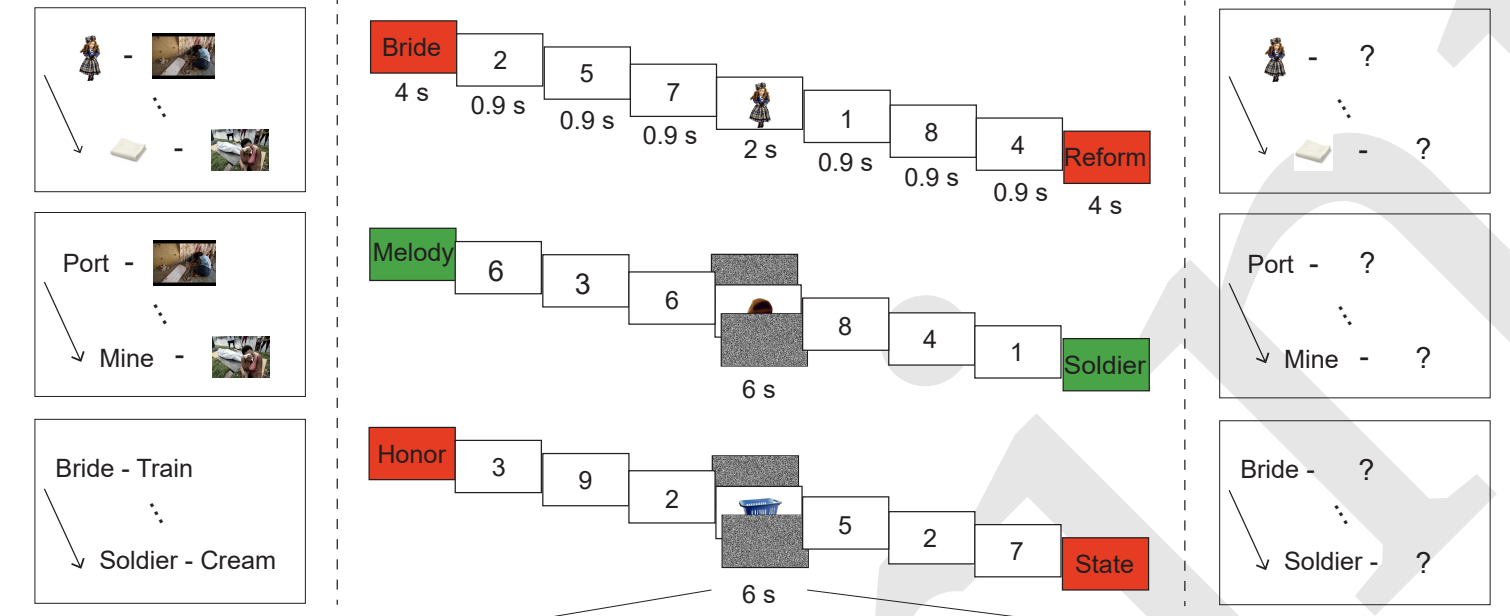

B

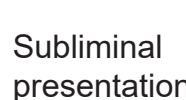

Full presentation sequence

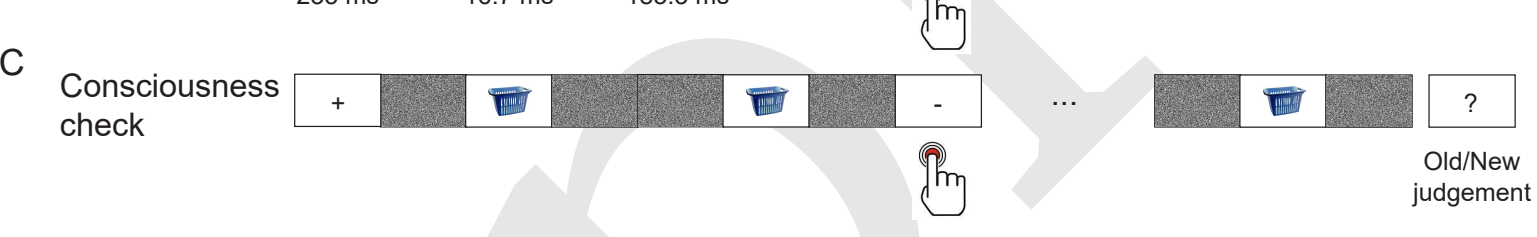

Figure 1. Experimental procedure. (A) In both experiments, subjects learned three series of cue-target associations with the first two series constituting the bystander pairs (in series 1 , the scenes were linked to object cues; in series 2 , the same scenes were linked to word cues) and the third series, the TNT pairs. Subjects then performed trials involving retrieval (Think, shown in green color) or retrieval suppression (No-think, shown in red color) on TNT pairs. Inserted between every two Think or No-think trials were repeated presentations of a "Bystander" object cue, from one of the Bystander pairs. Think Bystanders and half of the No-think Bystanders were reactivated subliminally with a masking procedure (middle section, A); Bystanders between the other half of the No-think trials were presented supraliminally for $2 \mathrm{~s}$ (middle section, top). Subjects covertly made an old/new judgment on the supraliminally presented bystander objects and performed a memory-irrelevant task (see B) during subliminal presentations. Even/odd buffer judgements on numbers were performed before and after Bystander cues to match the immediate task context surrounding bystander items across Think and No-think trials. The amnesic shadow and the suppression-induced forgetting effects were assessed at the end, in which the Bystander cues and then the TNT cues appeared and participants reported the corresponding targets (panel A, right side). (B) Procedure for the subliminal reactivation. The whole series, which lasted $6 \mathrm{~s}$, involved a fixed procedure which contained six repetitions of the following events: a 233 ms fixation cross, four 183.3 ms white noise masks, and two 16.7 ms cue pictures. Occasionally, the fixation cross would change to a horizontal/vertical line and subjects detected the change by key pressing. (C) Consciousness check at the end of the experiment. The consciousness check used the same subliminal presentation procedure as in the Think/No-think phase. The only difference was that after each trial, subjects instead judged whether they could identify the masked cue object, and whether the object was old or new in the experiment.

sis, the overall recognition accuracy in the Consciousness 14 Checking task in the final sample was $50.30 \%$ (Table 1 ), which was not different from the chance level of 50\%. The d' of the old/new recognition was -1.43 , indicating that subjects could not recognize the masked stimuli. We note that this exclusion standard is conservative in that participants in the consciousness checking phase were directed to intentionally identify and recognize masked objects as their main task, which they were not asked to do in the earlier TNT phase.

Having eliminated participants who could have identified the subliminal bystander items, we then tested whether unconscious Bystander reactivation led to an am- 2 nesic shadow. We conducted a 2 (cue type: trained cue vs. independent cue) $\times 4$ (suppression status: Think, No-think conscious, No-think unconscious, and Control) repeated measures ANOVA on both the identification and gist accuracy of the bystander targets (Figure 2B) separately. For identification accuracy, the main effects of suppression status $\left(\mathrm{F}(3,117)=6.16, \mathrm{p}<.001, \eta_{\mathrm{p}}{ }^{2}=0.14\right)$ and cue type $\left(\mathrm{F}(1,39)=28.36, \mathrm{p}<.001, \eta_{\mathrm{p}}{ }^{2}=0.42\right)$ were both significant. The two factors did not interact $(\mathrm{F}(3,117)$ $=1.27, \mathrm{p}=.29, \eta_{\mathrm{p}}{ }^{2}=0.03$ ), confirming similar amnesic shadow effects, irrespective of whether we tested people with the cue used to reactivate the Bystander scene or not. Considering that the trained and independent cues 

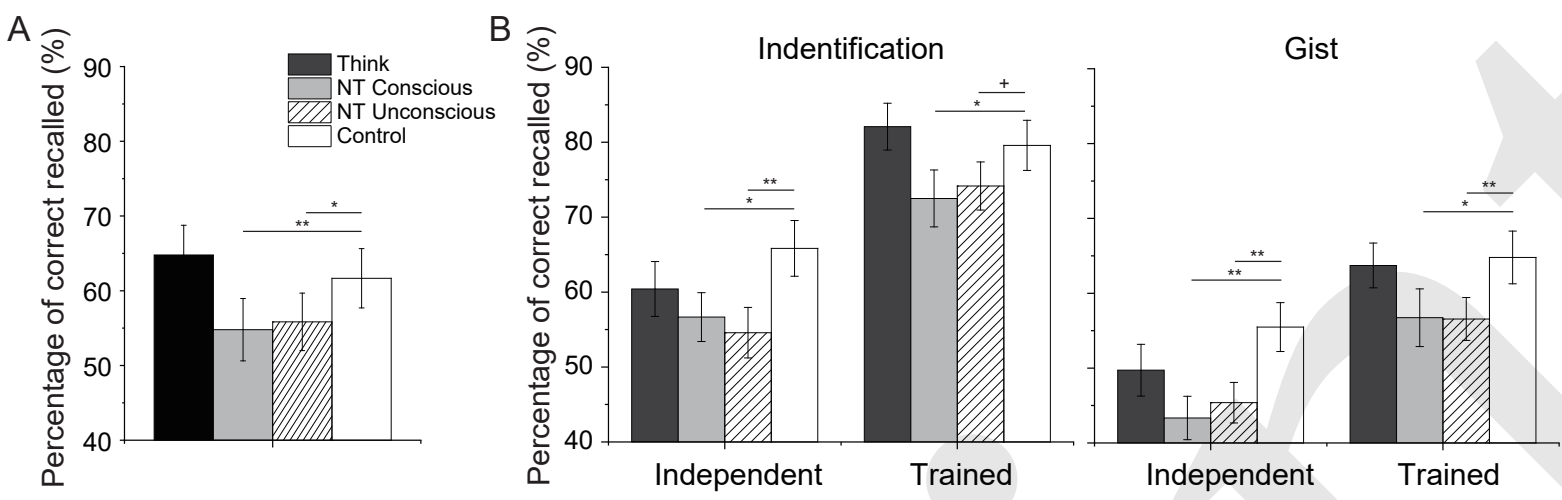

Figure 2. Suppression- and shadow-induced forgetting from Experiment 1. (A) Percentage of targets recalled for the Think/No-think (TNT) pairs Voluntary suppression consistently disrupted recall performance, showing suppression-induced forgetting in the conscious and unconscious No-think conditions. (B) Percentage of bystander images identified (left) and percentage of gist information recollected (right) for the two sets of bystander pairs. Both conscious and unconscious memory reactivation with the amnesic shadow induced by No-think trials impaired later recall of Bystander Scenes. Asterisks represent significant differences $\left(+\mathrm{p}<.06\right.$; ${ }^{*} \mathrm{p}<.05,{ }^{* *} \mathrm{p}<.01$, Two-tailed $\mathrm{t}$ test). Error bars indicate standard errors of the mean.

received different treatments - with the trained cues ex- 3 posed during the shadow period and the independent cues, not-we tested the effect of each cue type separately. We found a significant main effect of suppression status for both the independent- $\left(\mathrm{F}(3,117)=3.87, \mathrm{p}=.011, \eta_{\mathrm{p}}{ }^{2}=\right.$ $0.09)$ and trained-cue tests $(\mathrm{F}(3,117)=4.10, \mathrm{p}=.008$, $\eta_{\mathrm{p}}{ }^{2}=0.10$ ). Replicating our previous findings, supraliminally reactivating bystanders within the amnesic shadow led to robust memory impairment, relative to Control items the cues for which were not re-exposed and this memory disruption arose irrespective of whether participants were tested with the re-exposed (trained cue) or the independent cue (independent-cue retrieval: $t(39)=$ -2.68, $\mathrm{p}=.011$, Cohen's $\mathrm{d}=0.42$; trained-cue retrieval: $\mathrm{t}(39)=-2.43, \mathrm{p}=.020$, Cohen's $\mathrm{d}=0.38)$. Critically, this amnesic shadow effect also occurred for subliminally reactivated bystanders (independent-cue retrieval: $t(39)=$ $-3.21, \mathrm{p}=.003$, Cohen's $\mathrm{d}=0.51$; trained-cue retrieval: $\mathrm{t}(39)=-1.96, \mathrm{p}=.057$, Cohen's $\mathrm{d}=0.31$ ). Interestingly, shadow-related forgetting did not differ reliably in magnitude between the conscious and unconscious conditions (ps $>.50$ ). In contrast, re-activating Bystander scenes between two Think trials did not reliably affect memory performance for the Bystanders, relative to memory for Control items (independent-cue retrieval: $\mathrm{t}(39)=1.45$, $\mathrm{p}=.156$, Cohen's $\mathrm{d}=0.23$; trained-cue retrieval: $\mathrm{t}(39)=$ $0.78, p=.438$, Cohen's $d=0.12$ ). Thus, participants' later ability to recall an aversive scene significantly declined when that scene had been cued subliminally in the window between two unrelated retrieval-suppression trials, consistent with an unconscious amnesic shadow effect.

For Gist accuracy, the same 2 by 4 repeated measures ANOVA revealed significant main effects for suppression status $\left(\mathrm{F}(3,117)=6.71, \mathrm{p}<.001, \eta_{\mathrm{p}}{ }^{2}=0.15\right)$ and cue type $\left(\mathrm{F}(1,39)=15.88, \mathrm{p}<.001, \eta_{\mathrm{p}}{ }^{2}=0.29\right)$. When inspecting the Trained and Independent Cue performance separately, both showed a robust main effect of suppression status (independent-cue retrieval: $\mathrm{F}(3,117)=5.02$, $\mathrm{p}=.003, \eta_{\mathrm{p}}{ }^{2}=0.11$; trained-cue retrieval: $\mathrm{F}(3,117)=$ 4.26, $\mathrm{p}=.007, \eta_{\mathrm{p}}{ }^{2}=0.10$ ). In line with the findings in the identification measure, both supraliminal memory reactivation (independent-cue retrieval: $\mathrm{t}(39)=-3.67$, $\mathrm{p}<.001$, Cohen's $\mathrm{d}=0.58$; trained-cue retrieval: $\mathrm{t}(39)=$ $-2.46, \mathrm{p}=.019$, Cohen's $\mathrm{d}=0.39$ ) and subliminal reactivation (independent-cue retrieval: $\mathrm{t}(39)=-3.10, \mathrm{p}=.004$, Cohen's $\mathrm{d}=0.49$; trained-cue retrieval: $\mathrm{t}(39)=-2.87$, $\mathrm{p}=.007$, Cohen's $\mathrm{d}=0.45$ ) within the amnesic shadow impaired later recall of the reactivated bystander scenes, compared to recall for Control items that were not reactivated. These effects occurred regardless of whether scenes were recalled from trained- or independent-cues, illustrating that memory for the scene was disrupted, independent of the cue used. Therefore, subliminally presenting an unwanted emotional memory within the amnesic shadow window impaired people's ability to recall key details related to the scene's meaning.

\section{The amnesic shadow effect was independent of subjective consciousness.}

Objective consciousness analysis based on post hoc selection suffers from problems such as regression to the mean, so we checked subjects' subjective consciousness. Although objective recognition accuracy was at the chance level on our Consciousness Check, subjects still reported identifying masked items occasionally. Overall, 23.96\% of the bystander cues (Table 1, 1.53 out of the 6 Think bystanders and 1.35 out of the 6 No-think unconscious bystanders) were reported to be visible. However, when asked whether the putatively identified items were previously studied, the old/new recognition accuracy for the "consciously" perceived items that were previously studied (i.e., old items) was only 76\% (Table 2). This suggests that participants may have been adopting a liberal strategy in reporting consciousness of the masked scene, sometimes reporting visibility when there was none. If so, this liberal strategy would imply that unreported items are likely not 
Table 1. Percentage of items that participants claimed to see (\%)

\begin{tabular}{lcccc}
\hline & Overall & Think & No-think & New \\
\hline Experiment 1 & 21.56 & 25.42 & 20.50 & 19.17 \\
Experiment 2 & 12.62 & 18.06 & 14.93 & 4.86 \\
\hline
\end{tabular}

Table 2. Old/New recognition accuracies for items participants claimed to see or to not see (\%))

\begin{tabular}{lcccccc}
\hline & \multicolumn{3}{c}{ Reported Seen } & \multicolumn{3}{c}{ Reported Unseen } \\
\hline & Think Old & No-think Old & New & Think Old & No-think Old & New \\
\hline Experiment 1 & 75.93 & 76.67 & 21.59 & 28.63 & 30.38 & 68.84 \\
Experiment 2 & 95.30 & 87.00 & 69.10 & 30.00 & 26.10 & 73.00 \\
\hline
\end{tabular}

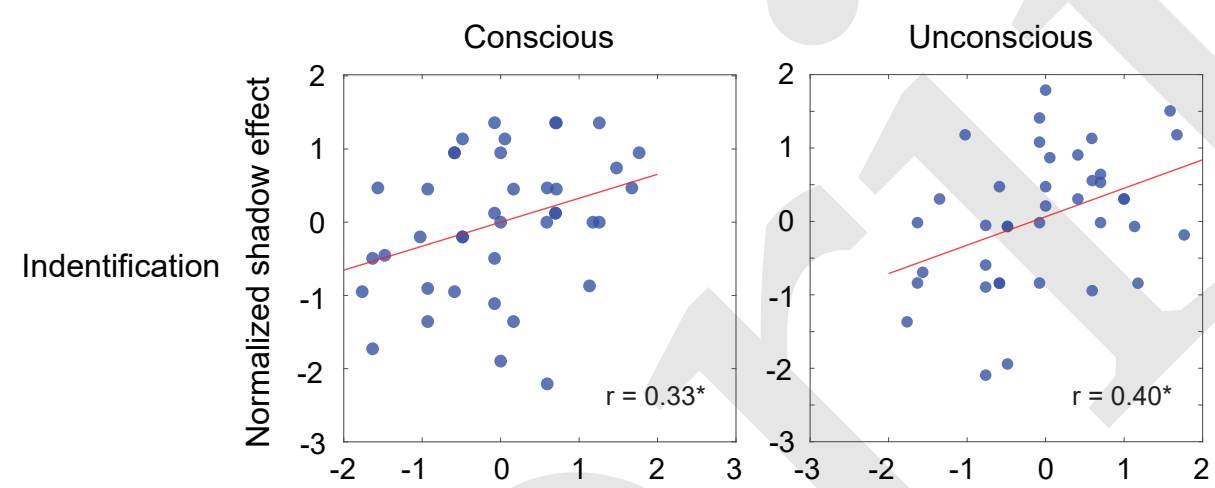

Figure 3. Correlation between the suppression- and shadow-induced forgetting effects. (Top) The degree of suppression-induced forgetting on TNT pairs predicted the shadow effect in the conscious (left) and unconscious (right) condition on the identification measure. (Bottom) The correlation was not detected for the Gist measure. * denotes significant correlations.

perceived. Building on this possibility, we tested whether the amnesic shadow effects would remain even when we excluded all bystander items reported visible during the Consciousness Check phase.

On average, 1.35 out of the 6 unconscious No-think bystanders were excluded. For the remaining items that could not be identified during the Consciousness Check, identification was still disrupted by the amnesic shadow under the independent-cue retrieval (No-think unconscious vs. Control: $\mathrm{t}(39)=-2.53, \mathrm{p}=.015$, Cohen's $\mathrm{d}=$ 0.40 ), though not under the trained-cue retrieval (Nothink unconscious vs. Control: $\mathrm{t}(39)=-1.49, \mathrm{p}=.145$, Cohen's $d=0.23$ ). We observed a robust amnesic shadow effect on our Gist measure on both independent- (No-think unconscious vs. Control: $\mathrm{t}(39)=-2.45, \mathrm{p}=.019$, Cohen's $\mathrm{d}=0.39)$ and trained-cue (No-think unconscious vs. Control: $\mathrm{t}(39)=-2.49, \mathrm{p}=.017$, Cohen's $\mathrm{d}=0.39$ ) tests. These findings provide converging evidence for an unconscious amnesic shadow: even when we restricted analyses to only those items that people couldn't consciously identify when they were intentionally trying, retrieval suppression disrupted bystander memories that were reactivated close in time.

\section{Suppression-induced forgetting predicts the amnesic shadow} effect.

Because retrieval suppression triggers both hippocampal down-regulation and SIF, the magnitude of SIF may be related to the amnesic shadow (Hulbert et al., 2016). We tested whether SIF on the TNT pairs predicted the amnesic shadow effect on the bystander scenes. We performed a 
Pearson correlation between the SIF effect (i.e., Control - 58 No-think) and its shadow effect on bystanders (i.e., Con- 5 trol - No-think) on both the trained- and independentcue tests using a robust statistical approach as described by (Pernet et al., 2013). Both effects were z-normalized within each item counterbalancing condition to account for item-effects, as in prior work (Anderson et al., 2004; Benoit et al., 2016; Hulbert \& Anderson, 2018). Consistent with our hypothesis, on the identification measure, SIF correlated with the overall shadow effect (averaged over the Trained and Independent Cues) in the No-think conscious condition (Figure 3 left, r-skipped $=0.33$, [0.07, 0.57] bootstrapped $95 \% \mathrm{CI}$ ). A significant correlation also was observed for the No-think unconscious condition after eliminating potential conscious items based on the Consciousness Check performance (Figure 3 right, r-skipped $=0.40,[0.15,0.60]$ bootstrapped 95\% CI). However, the same correlation was not detected in the Gist measure for either the conscious (r-skipped $=0.26,[-0.04,0.57]$ bootstrapped $95 \% \mathrm{CI}$ ) or the unconscious condition ( $\mathrm{r}-$ skipped $=0.09,[-0.24,0.38]$ bootstrapped $95 \% \mathrm{CI}$ ). Overall, successful SIF was linked to both the conscious and unconscious shadow effect, despite the word pairs used in the TNT and bystander scenes being entirely unrelated to one another, consistent with the possibility that retrieval suppression had set in motion processes that disrupted scene retention.

\section{Experiment 2}

The findings of Experiment 1 suggest that unconsciously reactivating a memory during the amnesic shadow induces significant forgetting. This conclusion assumes, however, that our offline consciousness test identified all items that participants had consciously perceived during the earlier amnesic shadow periods. However, participants in Experiment 1 might have been able to report the masked items if we had simply asked them to do so immediately during the amnesic shadow period. To exclude this possibility, Experiment 2 adopted a maximally sensitive trial-by-trial online consciousness check to probe for awareness of the item immediately upon its presentation.

In this new online procedure, participants judged their consciousness state for every item. During the subliminal bystander exposures, participants pressed a button to indicate immediately whether they could consciously identify the item; if so, they verbally reported what they saw. This procedure eliminates doubt about whether a given exposure might have been perceived. In addition, after the full No-Think trial had ended, a question mark prompted participants to judge whether the item they had identified was old or new (Figure 4). To ensure that the answer to the latter episodic recognition judgments was not always "yes", we included novel foils trials (hereinafter called "novel" trials). During these trials, instead of presenting a studied bystander cue, we subliminally exposed an entirely novel cue object. Because the main goal of Experiment 2 was to firmly establish the subliminal nature of the amnesic shadow effect, we eliminated the supraliminal condition.
To further bolster confidence that the items were truly unconscious, we adopted strict subject and item exclusion criteria based on our indices of conscious awareness.

\section{Suppression induced forgetting on TNT pairs.}

First, we verified that suppression-induced forgetting occurred, despite our introduction of an online consciousness checking task. Replicating prior work, recall accuracy for TNT pairs varied significantly across the four conditions (i.e., the Think, No-think unconscious-old Nothink unconscious-novel, and Control conditions) (Fig. $\left.2 \mathrm{~A}, \mathrm{~F}(3,141)=11.97, \mathrm{p}<.001, \eta_{\mathrm{p}}{ }^{2}=0.20\right)$. Critically, retrieval suppression impaired recall performance for NoThink items compared to that observed for Control items: Significant SIF arose regardless of whether intervening bystander exposures presented previously studied cues (i.e. "old cues; No-think unconscious old vs. Control: $t(47)=-2.15, p=.037$, Cohen's $d=0.31$ ) or new foil cues (i.e. "novel" cues; No-think unconscious novel vs. Control: $\mathrm{t}(47)=-2.60, \mathrm{p}=.013$, Cohen's $\mathrm{d}=0.37$ ), confirming that suppression-induced forgetting occurred. In contrast, retrieval of items during Think trials increased recall performance (Think vs. Control: $\mathrm{t}(47)=2.82, \mathrm{p}=.007$, Cohen's $\mathrm{d}=0.41)$.

\section{Subliminally presented bystander cues were mostly} unconscious.

Next, we used the findings from our trial-by-trial online consciousness check to quantify the extent to which participants were conscious of the subliminally reactivated bystander cues. Participants failed to identify the subliminally presented item on $87.38 \%$ of the items, on this task. Thus, only $12.62 \%$ of the subliminal presentation items were reported visible and correctly named (Table 1). We further examined the episodic recognition accuracy of reported items (as measured at the end of each trial) and found high accuracy for items reported to be consciously perceived $90 \%$ (Table 2). After eliminating the $12.62 \%$ of the items that participants identified, recognition performance for the remaining $87.38 \%$ of bystanders was exceptionally low $(43.02 \%)$ and indeed lower than chance $(\mathrm{t}(47)=-5.49, \mathrm{p}<.001$, Cohen's $\mathrm{d}=0.79)$. To the extent that the main determinant of conscious awareness is the introspective judgment that we are aware of a stimulus (Cleeremans, 2011), our findings indicate that the remaining subliminally presented items were truly unconscious.

\section{The amnesic shadow impairs unconsciously reactivated bystander memories.}

Having removed items that were consciously perceived, we next calculated the critical amnesic shadow effect on unconscious bystanders. We performed a 2 (cue type: trained cue vs. independent cue) $\times 3$ (suppression status: Think-unconscious, No-think-unconscious, and Control) repeated measures ANOVA separately on the identification and gist accuracy of bystander target recall performance (Figure 5B). For the identification accuracy measure, bystander recall varied due to our manipulation as reflected in a main effect of suppression status $(\mathrm{F}(2,94)=4.79$, 


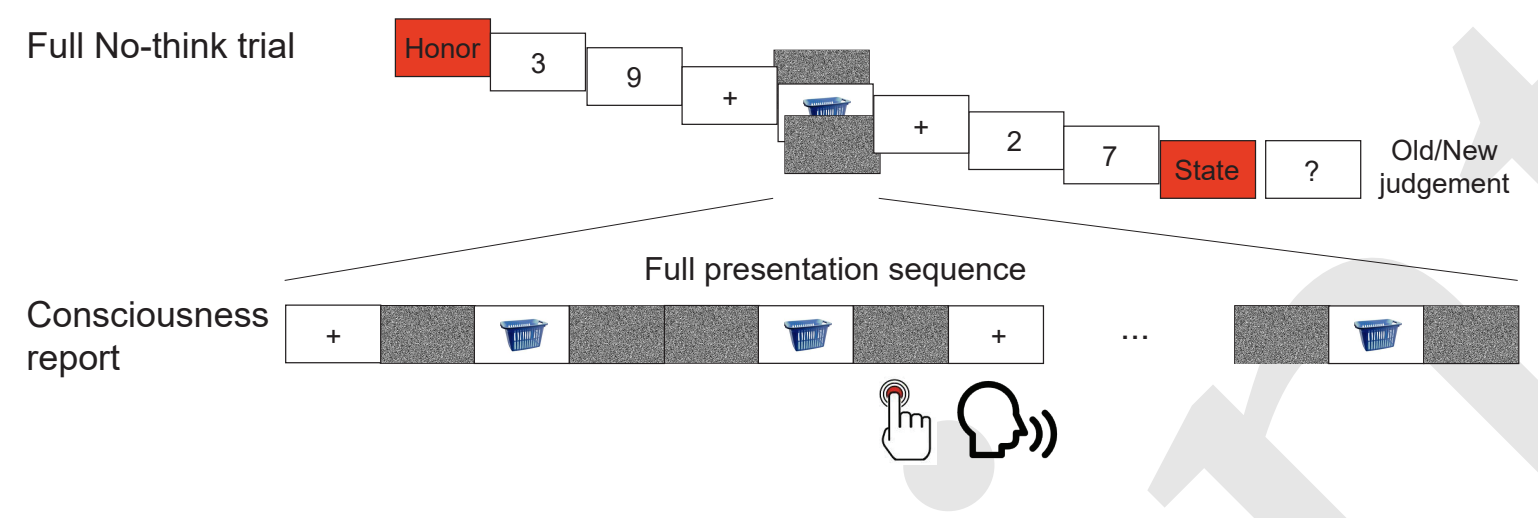

Figure 4. The online consciousness check procedure used in Experiment 2. We inserted the subliminal reactivation of bystanders between two TNT trials (No-think trials in the figure). The whole series, which lasted 6 s, involved a fixed procedure which contained six repetitions of a 233 ms fixation cross, four $183.3 \mathrm{~ms}$ white noise masks, and two $16.7 \mathrm{~ms}$ cue pictures. Participants judged whether they could identify the cue object by a key press and verbally reported the content of the picture. At the end of the full trial, a question mark appeared, prompting participants to judge whether the object they been exposed to was old or new.

$\left.\mathrm{p}=.010, \eta_{\mathrm{p}}{ }^{2}=0.09\right)$. Although overall recall varied across our two cue types $\left(\mathrm{F}(1,47)=16.74, \mathrm{p}<.001, \eta_{\mathrm{p}}{ }^{2}=0.26\right)$, cue-type did not interact with suppression status $(\mathrm{F}(2,94)$ $\left.=0.24, \mathrm{p}=.787, \eta_{\mathrm{p}}^{2}=0.01\right)$, confirming similar amnesic shadow effects under our trained and independent cues. Based on our a priori prediction of shadow-induced forgetting, we compared recall in the No-think-unconscious condition with that of the Control condition. Supporting our central hypothesis, and replicating Experiment 1, we found significant forgetting on the independent cue test: No-Think bystanders were recalled more poorly than were Control bystanders ( $\mathrm{t}(47)=-2.74, \mathrm{p}=.009$, Cohen's $\mathrm{d}=$ 0.39 ) as also more poorly than were Think bystanders $(\mathrm{t}(47)=-2.01, \mathrm{p}=.051$, Cohen's $\mathrm{d}=0.29)$. Although not significant, we detected a trend of shadow-induced forgetting in trained-cue retrieval $(\mathrm{t}(47)=-1.94, \mathrm{p}=.059$, Cohen's $d=0.28$ ). We found no memory improvement for Think bystanders (independent-cue retrieval: $\mathrm{t}(47)=$ $-0.48, \mathrm{p}=.637$, Cohen's $\mathrm{d}=0.07$; trained-cue retrieval: $\mathrm{t}(47)=-0.61, \mathrm{p}=.543$, Cohen's $\mathrm{d}=0.09)$.

For the gist accuracy, the same 2 by 3 repeated measures ANOVA revealed significant main effects for suppression status $\left(\mathrm{F}(2,94)=11.24, \mathrm{p}<.001, \eta_{\mathrm{p}}{ }^{2}=0.20\right)$ and cue type $\left(\mathrm{F}(1,47)=10.05, \mathrm{p}=.003, \eta_{\mathrm{p}}{ }^{2}=0.18\right)$. In line with the findings from the identification measure, subliminal memory reactivation within the amnesic shadow window induced by No-Think trials consistently impaired the reactivated bystanders, when compared with recall in the Control condition (independent-cue retrieval: $\mathrm{t}(47)=-$ 3.82, $\mathrm{p}<.001$, Cohen's $\mathrm{d}=0.55$; trained-cue retrieval: $\mathrm{t}(47)=-3.29, \mathrm{p}=.002$, Cohen's $\mathrm{d}=0.48)$ and also when compared to recall for Think bystanders (independentcue retrieval: $\mathrm{t}(47)=-2.01, \mathrm{p}=.050$, Cohen's $\mathrm{d}=0.29$; trained-cue retrieval: $\mathrm{t}(47)=-2.39, \mathrm{p}=.021$, Cohen's $\mathrm{d}$ $=0.34$ ). The findings of Experiment 2 thus confirmed that both coarse and detailed information about unwanted memories could be disrupted simply by subliminally presenting reminders to those events in the amnesic shadow window induced by the suppression of an independent memory.

\section{Analysis of the unconscious amnesic shadow effect across two experiments.}

To confirm the unconscious amnesic shadow effect, we removed the subjectively perceived unconscious bystanders for each participant and combined the participants from Experiments 1 and 2. This analysis strongly affirms that the amnesic shadow disrupted the identification and gist recall for unconsciously reactivated bystander memories (Figure 6). The shadow-induced forgetting was most robust when memory for bystanders was tested with the independent-cue retrieval (Identification: $\mathrm{t}(87)=-3.74$, $\mathrm{p}<.001$, Cohen's d = 0.40; Gist: $\mathrm{t}(87)=-4.44, \mathrm{p}<.001$, Cohen's $d=0.47$ ), but also was significant when memory was tested with the trained-cue that was subliminally exposed during the shadow period (Identification: $\mathrm{t}(87)=$ -2.44, $\mathrm{p}=.017$, Cohen's $\mathrm{d}=0.26$; Gist: $\mathrm{t}(87)=-4.13$, $\mathrm{p}<.001$, Cohen's $\mathrm{d}=0.44)$. Overall, the amnesic shadow induced by retrieval suppression on a neutral memory is clearly sufficient to disrupt an independent emotional memory that was subconsciously reactivated close in time.

In Experiment 1, we observed a correlation between the suppression-induced forgetting effect for our neutral word pairs and the amnesic shadow effect for bystanders in the conscious condition. But the same correlation was weak for items that were subliminally exposed. We speculated that this might arise because the amnesic shadow effect relies not only on the suppression of the hippocampal functions (induced by retrieval suppression on NT pairs) but also on how effectively our bystanders were reactivated by their cues, an outcome that should determine its reliance on the hippocampal functions. Variability in the strength of reactivation created by bystander cues might reduce the correlation between suppression-induced forgetting and the amnesic shadow effect. To increase the power of the correlation analysis, we combined participants from 

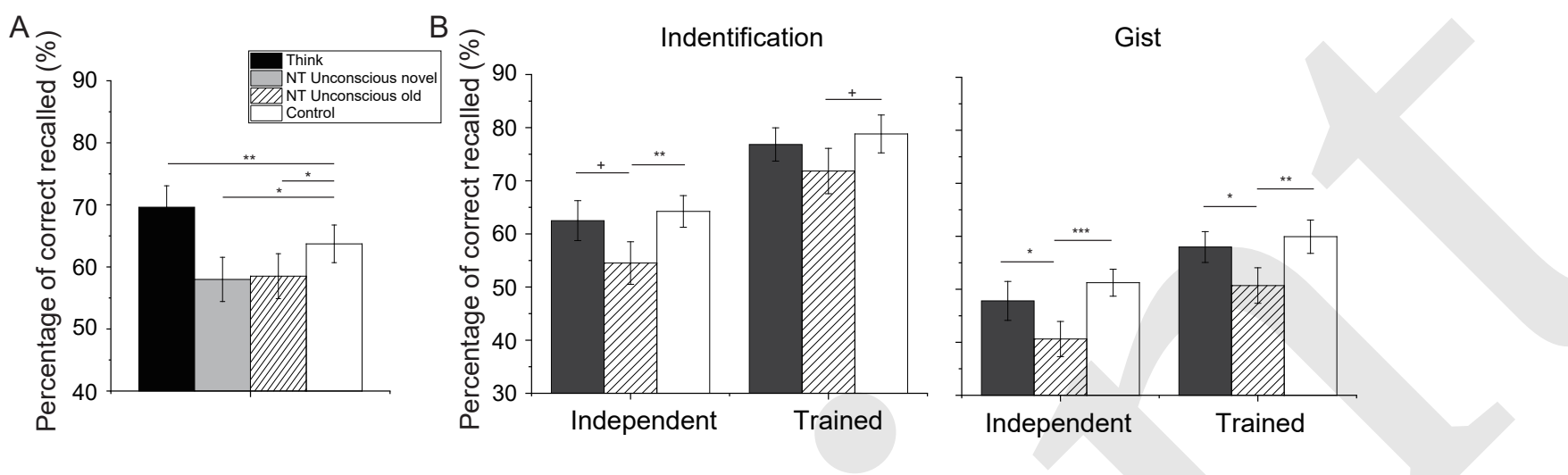

Figure 5. Results from Experiment 2. (A) Percentage of targets recalled for the Think/No-think (TNT) pairs. Retrieval suppression consistently disrupted recall performance, causing suppression-induced forgetting in the unconscious No-think condition. This suppression-induced forgetting effect was unaffected by whether cues to old or novel bystanders were exposed during the shadow period for No-Think trials. (B) Percentage of bystander images that were recalled according to our identification (left) and Gist measures (right) on the Trained and Independent probe tests. Unconscious cue exposure between two No-think trials caused forgetting of the reactivated memories linked to those cues. Asterisks represent significant differences $(+\mathrm{p}<.06 ; * \mathrm{p} .05, * * \mathrm{p}<.01, * * * \mathrm{p}<.001$, Two-tailed t test.). Error bars indicate standard errors of the mean.

the two experiments. Indeed, with this larger sample size, on our identification measure, suppression-induced forgetting correlated with the amnesic shadow effect on unconsciously reactivated bystanders (Figure 6B, r-skipped $=$ $0.32,[0.09,0.48]$ bootstrapped $95 \%$ CI). However, the same correlation was not significant when we examined the gist measure (r-skipped $=0.04,[-0.18,0.24]$ bootstrapped $95 \% \mathrm{CI}$ ). Together, these findings suggest that, as with consciously reactivated bystanders, unconsciously re- 4 activated memories show amnesic shadow effects that are linked to the efficacy of retrieval-suppression processes, as reflected in suppression-induced forgetting.

\section{Discussion}

Our findings show that unpleasant events can be forgotten by simply cuing them unconsciously during a time window in which hippocampal function has been suppressed. We took advantage of recent finding showing that suppressing retrieval disrupts hippocampal function, inducing anterograde and retrograde amnesia for unrelated events encoded near in time to suppression (Hulbert et al., 2016). Building on this amnesic shadow finding, we reasoned that if people suppressed entirely neutral materials (e.g., simple neutral word pairs), it should induce forgetting of 60 upsetting events reactivated subliminally within the am- 6 nesic shadow period. Our findings strongly confirm this unconscious shadow-induced forgetting prediction. Importantly, shadow-induced forgetting didn't occur only for the reminder cues that we subliminally re-exposed; rather, the subliminally reactivated memory was also less acces- 6 sible when tested with an independent cue that was not subliminally re-exposed. This cue-independent forgetting indicates that the memory itself suffered generalized forgetting that arose from its reactivation during the shadow period. Thus, we induced people to forget an unpleasant memory without ever being consciously aware of the reminders that triggered the forgetting, or any intention to forget these experiences.

To ensure that the amnesic shadow effect was entirely unconscious, our two experiments employed strict controls to exclude conscious items. Experiment 1 used an offline awareness test whereas Experiment 2 adopted an online trial-by-trial awareness test, both with subjective ratings and objective recognition indices. In Experiment 1, we ensured that performance on the forced-choice recognition tests at the individual subject and group levels were at the chance level. On that basis, we excluded items that were subjectively reported as visible. To obtain an assessment of consciousness, more directly tied to individual trials, Experiment 2 asked participants to indicate, on every trial, awareness immediately as they experienced it, and to report the content of the conscious item if one was experienced. This manipulation proved remarkably effective at isolating true awareness, as the reported visible items were nearly perfectly recognized on the recognition test (Table 1). Our two experiments consistently observed the shadow-induced forgetting effects in the unconscious condition, even after excluding all subjectively reported conscious items. As such, awareness of reminders appears to not be a prerequisite for shadow-induced forgetting to happen.

The ability to induce forgetting without participants awareness of the re-exposure process provides potentially significant clinical and theoretical advantages. These advantages flow directly from our use of the amnesic shadow phenomenon to create a window of vulnerability during which memories can be disrupted. Clinically, the most significant advantage lies with ability to induce an adverse hippocampal state by suppressing retrieval of an entirely unrelated, emotionally neutral memory. Thus, the focus of the retrieval suppression intervention can be on entirely benign stimuli that are not related to or in the same format as the bystanding target memory. This generalization property enables emotional memories to be modulated 

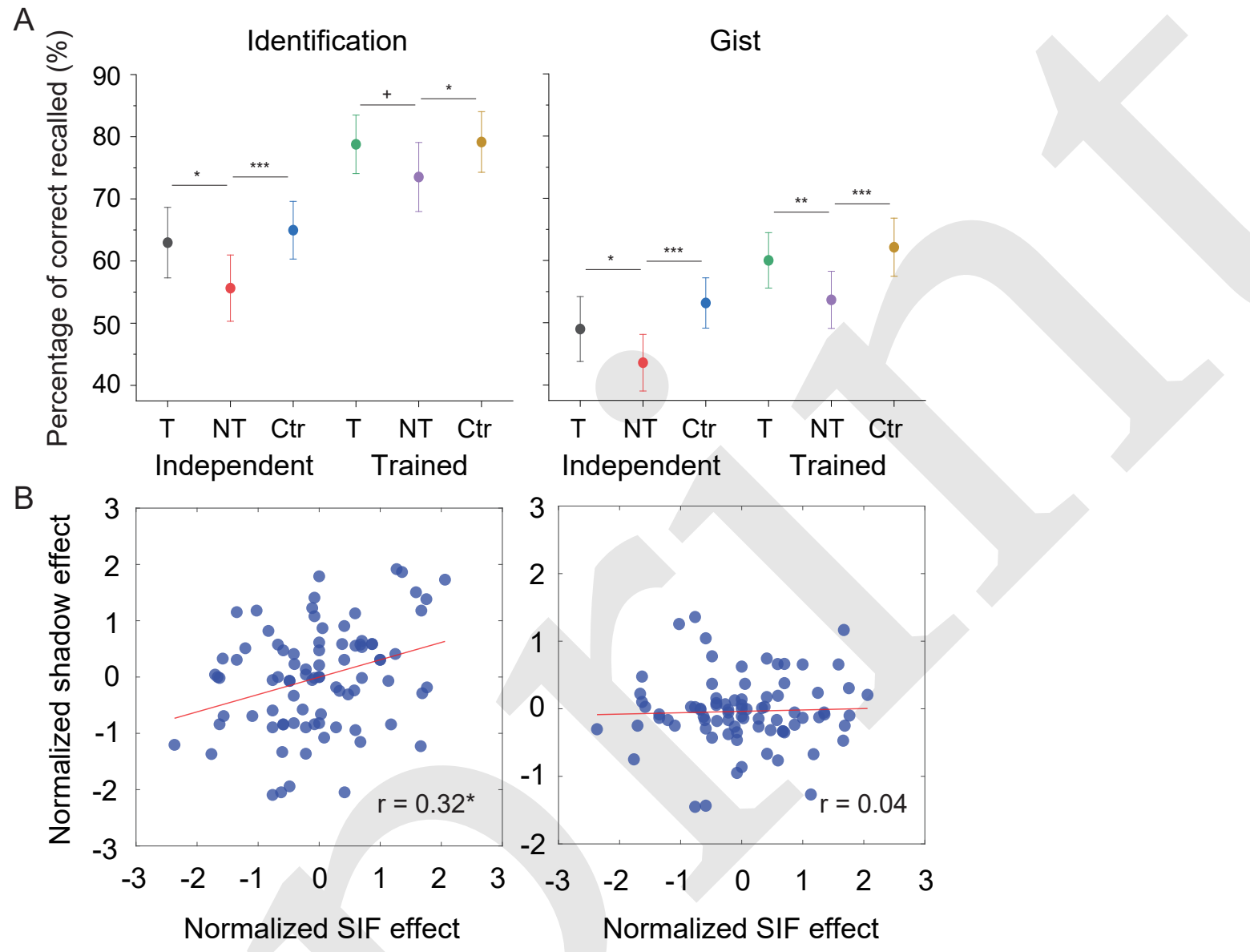

Figure 6. Combined Analyses of Experiments 1 and 2. (A) Percentages of bystander images identified (left) and gist information recalled (right) for bystander pairs recalled on the Independent or Trained Probe tests (T, Think; NT, No-think; Ctr, Control). Asterisks represent significant differences $(+\mathrm{p}<.06 ; * \mathrm{p} .05, * * \mathrm{p}<.01, * * * \mathrm{p}<.001$, Two-tailed t test.). Error bars indicate $95 \%$ confidence interval. (B) Correlation between the suppressionand shadow-induced forgetting effects combining across the two experiments. (Left) The degree of suppression-induced forgetting on TNT pairs predicted the shadow effect in the identification measure. (Right) The degree of suppression-induced forgetting did not predict the shadow effect on the gist recall measure. * denotes significant correlations.

without perceptual awareness. Thus, our findings provide 22 a novel method capable of bypassing the unpleasantness of 23 consciously re-exposing people to unwelcome content, as 24 occurs in conventional psychotherapeutic treatments for ${ }_{25}$ trauma-related psychiatric disorders. Theoretically, subliminal reactivation of target memories provides other advantages. Because participants were entirely unconscious to the interpolated bystander cues, our procedure rules out theories that might attribute the amnesic shadow to demand characteristics. One might hypothesize, however, implausible, that participants learn to withhold the recall of some bystanding memories on the final test, based on their conscious association of each item to adjacent trials, and assumptions about what the experimenter wanted. This possibility is entirely excluded in our unconscious reactivation method: because participants could no longer realize the identity of each bystander cue, it is impossible for them to adopt such a strategy. Our findings that subliminally reactivated bystander memories are disrupted by the amnesic shadow is thus free of the influence of the experimenter effects (Kennedy \& Taddonio, 1976).
Despite the advantages discussed above, several issues must be examined before clinical application is considered. First, because the amnesic shadow is induced by inhibitory control over hippocampal activity (Hulbert et al., 2016; Zhu \& Wang, 2021), the forgetting effect on unconsciously presented items may be restricted to hippocampus-dependent memories. To the extent that the stimuli involved in an unpleasant event have undergone affective conditioning, they may continue to evoke emotional responses even after shadow-induced forgetting occurs, given the reliance of affect conditioning on amygdala. Second, our masking procedure failed to block consciousness of Bystander pictures occasionally. Although the consciously perceived items can be eliminated from analyses to test our theoretical hypothesis, clinical applications demand even more thorough and effective masking procedure to ensure fully unconscious forgetting. A procedure that blocks consciousness while maximizing memory reactivation is preferred. Nevertheless, combining the current approach with other methods may exploit and magnify the strengths of each. Our unconscious forgetting pro- 
cedure could be combined with conventional treatments to produce synergistic forgetting effects and to prevent premature drop out of treatment. For example, applying our procedure before exposure therapy may help relieve the distress due to excessive traumatic memory intrusions, whilst providing an opportunity to extinguish conditioned emotional responses. In addition, recent studies have used neuroimaging and machine learning methods to develop unconscious neural reinforcement interventions that impacted physiological activity to feared stimuli (TaschereauDumouchel et al., 2018). Our procedure, could provide a strong complement to such a procedure, focused on the mitigation of intrusive episodic memories.

Whereas its effects are meant to be unconscious to participants, our subliminal reactivation procedure aims to activate the target memory rather than to prevent memory reactivations. This, in fact, is a critical prerequisite for the amnesic shadow to work. The amnesic shadow arises because retrieval suppression temporarily disrupts hippocampal function, rendering traces reactivated in this window vulnerable. Any memory changes to the subliminally exposed event, should only happen if the cued event recruits hippocampal functions. The predicted dependency of the amnesic shadow on hippocampal processing of bystander items motivated (Hulbert et al., 2016) use of episodic encoding, our prior use of episodic retrieval (Zhu \& Wang, 2021), and our current use of associative memory reactivation to induce forgetting. Notably, achieving robust mnemonic reactivation subliminally is challenging, because the degree of reactivation depends on a complex interaction between the cue stimulus and the masks. Variability in the success of subliminal reactivation might partly obscure the association between the SIF effect and the subliminal amnesic shadow effect. However, across our two experiments, we showed a significant correlation between the two effects, supporting a link between shadow-induced forgetting and retrieval suppression.

In conclusion, we have exploited the systemic impact of retrieval suppression on hippocampal functions to create an unconscious forgetting method. Our studies provide strong evidence that forgetting of affective memories can be achieved completely unconsciously and without direct experiencing of aversive content, simply by inserting reminders to them into the amnesic shadow induced by suppressing entirely independent and benign memories. Moreover, our subliminal reactivation procedure ensures the memory-altering features of our procedure can be tested in a double-blind manner, wherein neither the experimenter nor the participants need be aware of which memories should be disrupted. This method holds the potential to complement existing therapeutic interventions for treating trauma and reducing high dropout rates triggered by distressing intrusions. More broadly, our findings provide strong converging evidence for the view that retrieval suppression engages a mechanism that globally suppresses hippocampal encoding and stabilization processes, disrupting even those activated hippocampal traces that do not fully enter awareness.

\section{Methods}

\section{Experiment 1}

Participants

For both experiments, sample sizes were determined in advance via a power analysis on the amnesic shadow effect in our previous study using a similar procedure (see https://osf.io/5c2hf for the preregistration). The power analysis yielded a sample size of 40 adults. Participants (aged 19-22, 32 females) were required to have normal or corrected-to-normal vision. Two additional participants were excluded due to above-chance level recognition performance in the objective consciousness check as described in the Procedure section. Informed consent was obtained in accordance with procedures approved by the Human Subject Review Committee of the Shaanxi Normal University.

\section{Materials}

The stimuli included a set of verbal word-pairs to implement the TNT task, and a set of word-picture pairs to be used as bystander stimuli.

TNT Pairs. We constructed 48 critical TNT paired associates. Each TNT pair was composed of two 2-character Chinese words (e.g., "legend - reason"). Each of these words was neutral in valence, as established by subjective rating by 30 independent subjects and Xu et al., 2021. The cue and the target for each pair were semantically unassociated with each other, as established by agreement between the three experimenters.

Bystander Stimuli. We constructed a set of 48 pairs to test for amnesic shadow effects. Each bystander item was composed of a target picture and two cues with which it was paired (in the form of A-X and B-X, where X was the target picture). Specifically, we selected 24 object photos that we paired them with a word and a picture cue, yielding 48 cue-target pairings. The bystander target pictures were affective scenes from (Küpper et al., 2014). These pictures, originally taken from the International Affective Picture System and online sources, included themes such as physical and sexual assault, witnessing injuries and death, natural disasters, and serious accidents (Zhu \& Wang, 2021). One set of bystander cues used object pictures. To simulate natural situations associated with involuntary trauma recall, each object cue resembled an item embedded in its paired scene. The other set of bystander cues used neutrally-valenced 2-character Chinese words (Xu et al., 2021). These word cues were not related to the scene, as determined by the judgment of the three experimenters.

The two sets of cue-target pairs were studied and trained in separate lists. In the Think/No-think (TNT) phase, the object cues appeared embedded between TNT trials and serve as the Trained cues, whereas the word cue did not appear during the TNT phase, serving instead as independent cues. Items from the TNT pairs were semantically unassociated with cues or targets from the bystander pairs. We selected another 12 object pictures to use for the con- 
sciousness check task.

The TNT and bystander pairs were each divided into four subsets (TNT: 12 items per condition; Bystander: 6 items per group), which will be used in one of four con- 6 ditions: Think unconscious, No-think conscious, No-think unconscious, and Control. Each subset of TNT pairs was yoked to a fixed subset of bystander pairs (i.e., when subset 1 was in the NT condition, the yoked bystanders were in the NT condition). The assignment of TNT item sets (along with their bystander) to experimental conditions was counterbalanced across participants.

\section{Procedure}

Learning phase. Participants studied three sets of cuetarget pairs, including two bystander pair sets (where set one had the form A-X, and set two, B-X) and one TNT pair set. To ensure comparative memory strength for pairs within the same set and to avoid memory integration of pairs across different sets, the three sets were studied separately in a fixed order: the trained-cue bystander pair set first (object-scene pairs), the independent-cue bystander set second (the word-scene pairs), and the TNT pairs last (the word-word pairs).

First, the trained-cue bystander pair set (i.e., objectscene pairs) was studied. Twenty-four object-scene pairs were presented to participants one at a time, each for $3 \mathrm{~s}$ (interstimulus interval $=1 \mathrm{~s}$ ). Test-feedback cycles followed, in which each cue appeared alone for up to $5 \mathrm{~s}$ and participants judged whether they could retrieve the corresponding scene or not by pressing one of two keys. When a key was pressed or when the response window expired, the target scene appeared to the right side of the cue. Participants then reported whether they had retrieved the target picture correctly by pressing one of two keys within $5 \mathrm{~s}$. Pairs that were self-reported as correctly recollected were eliminated from the subsequent test-feedback cycles. Test-feedback cycles continued until all pairs were correctly recollected. Next, the independentcue bystander set (i.e., word-scene pairs) were studied, using the same procedure. To avoid integration of the two bystander sets, participants were informed that the target pictures would be the same as those in the first set. They were instructed to study the new set without thinking of the first set and to avoid thinking of the three items (i.e., two cues and one common target) together. Finally, the 48 TNT word pairs were studied, using the same procedure as for the bystander pairs.

TNT phase. Two critical manipulations - the Think/Nothink task and conscious/unconscious memory reactivation - were interleaved in this phase. The Think/No-think manipulation was performed on TNT word pairs. Our aim in using the TNT task was to induce the amnesic shadow using "No-Think" trials (discussed shortly). Conscious or unconscious memory reactivation was performed by presenting bystander cues within the amnesic shadow intervals during the TNT task.

From the 48 word-pairs that we trained during the learning phase, 36 pairs participated (12 pairs from each of three subsets). Each trial in this phase presented a single cue from one of the pairs for 4 seconds, which participants were instructed to view continuously. Cues from one of the subsets were presented in green (Think trials) and cues from the other two subsets were presented in red (No-think-conscious and No-think-unconscious trials). For Think trials, participants were instructed to recall the associated target word upon cue onset and to think of it silently for the full $4 \mathrm{~s}$. For No-think (conscious and unconscious) trials, participants were asked to avoid thinking about the associated target word while sustaining their attention on the cue word for the full 4-s duration. Procedurally No-Think-conscious and No-Think-unconscious trials did not differ during the No-Think task itself, but only differed by virtue of the Bystander task done in its vicinity (to be discussed next). For the No-Think task, the standard direct suppression instructions were used (Benoit \& Anderson, 2012). These instructions emphasized that participants should try to stop retrieval of the target word while avoiding replacing the target with any other diversionary thoughts or images (Wang et al., 2019). Cues from the fourth subset of trained pairs did not appear during the TNT phase. These pairs, which were learned at the same time as the Think and No-Think pairs served as a Control condition for the TNT manipulation, enabling us to estimate what final memory performance would be, given that neither retrieval nor suppression had been performed on pairs.

During the TNT task, we reactivated the target memories of three sets of bystander pairs by presenting their retrieval cues in between Think trials or between No-Think trials. Notably, only object cues were presented as bystanders (hereinafter referred to as Trained cues). No word cues for the relevant scenes appeared during the TNT phase; these word cues were independent of the TNT manipulation (hereinafter referred to as independent cues). The cues from the three bystander subsets underwent different manipulations. To examine the amnesic shadow effect, cues from one bystander subset were each presented between two Think trials and cues from the other two bystander subsets were each presented between two Nothink trials (No-think trials have been found to induce an amnesic shadow that is disruptive to memories reactivated close in time to them). To test the influence of consciousness on the amnesic shadow effect, the object cues from Think bystanders and one subset of No-think bystanders were presented subliminally. We used for subliminal memory reactivation procedure from (Degonda et al., 2005). Specifically, the object cue (S) was presented 12 times within $6 \mathrm{~s}$ for $16.7 \mathrm{~ms}$ (the total presentation duration was thus $2 \mathrm{~s}$ ). Each cue was forwardly and backwardly masked by a 183.3-ms white Gaussian noise mask (M). A 233-ms fixation cross (F) was presented 6 times within the $6 \mathrm{~s}$ separating every two series of M-S-M sequences. Overall, one trial contained six continuous repetitions of the stimulation sequence of F-M-S-M-M-S-M. The fixation cross (F) would occasionally change into a vertical/horizontal bar, and participants' task was to report its occurrence by 
pressing a key. Each of the items in the remaining subset of 59 No-think bystanders, which was used as a conscious com- 6 parison, was presented uninterrupted on the screen for 2 $\mathrm{s}$, during which participants were encouraged to covertly judge whether they had studied this cue object. Notably, when bystander cues appeared, participants were not instructed to retrieve or suppress the target scene picture in any condition. The fourth subset of the bystander pairs did not appear in the TNT phase; these pairs, which were learned at the same time in the learning phase, served as control condition that allowed us to estimate, on the later test, retention of pairs that had never been reactivated during the TNT phase.

Before and after each bystander, we inserted "buffer" intervals during which a series of 2-3 digits were presented on the screen. Participants classified each digit according to whether it was odd or even by pressing one of two keys. Each digit stayed on the screen for $0.9 \mathrm{~s}$ and was interleaved by a blank screen for $0.15 \mathrm{~s}$. This procedure ensured that the same task was performed before and after every bystander cue in all conditions; thus, bystanders embedded between two No-Think or two Think trials were nonetheless matched with regard to any task-set switching requirements before or after the bystander exposure (Hulbert et al., 2016; Zhu \& Wang, 2021), holding constant any interference such task transitions may cause. Taken together, the stimulation sequence for a full trial was "TNT task - buffer task - bystander task - buffer task - TNT task", followed by a 2-s fixation cross. Trials from different conditions appeared in a random order. Each TNT and bystander task repeated eight times in eight blocks. Because one bystander cue was embedded between two TNT cues, each bystander pair was paired with a fixed group of two TNT pairs in all repetitions.

Testing phase. Following the TNT phase, participants received two tests in a fixed order. A cued-recall test was used as in previous studies (Küpper et al., 2014; Zhu \& Wang, 2021), which presented the cues for participants to verbally report the content of the target scene within $15 \mathrm{~s}$ (interstimulus interval $=1 \mathrm{~s}$ ). First, we tested participants' memories for the bystander scenes, once using the object cue (trained cue) and once using the independent (word) cue, in separate blocks. We tested half of the participants with the trained cue first and the other half with the independent cue first. Next, we tested participants' memory for the TNT pairs. Each cue word appeared on the screen, one at a time, and participants wrote down its associated target. The test of TNT pairs was self-paced.

Consciousness check. At the end, we checked participants' consciousness level of the subliminally presented bystander cues. The 6 Think unconscious and the 6 Nothink unconscious bystanders were included along with 12 novel object pictures. To reproduce the unconscious bystander task, we used the same stimulation sequence of 6 repetitions of F-M-S-M-M-S-M and participants performed the same bar discrimination task as in the main experiment. After the sequence ended, participants reported whether they could see the content of the object
(S) that had appeared within the series and then made an old/new response on the object. Response time was not limited.

\section{Data analysis}

We performed the consciousness check analysis in two steps. First, we calculated the old/new recognition accuracy for the subliminally presented bystander items and foils for each participant. We then compared each participant's performance in the forced-choice test to the onetailed $5 \%$ cutoff $(66.7 \%)$ of the chance distribution of correct choices (Degonda et al., 2005). Two subjects exceeded this cutoff and were replaced. Second, for each of the remaining participants, we excluded all subjectively reported visible items.

For TNT pairs, the percentage of correctly recalled target items was calculated for each condition. For bystander pairs, scoring for the verbal descriptions of the target scene images was based on the criteria used in (Zhu \& Wang, 2021) and (Küpper et al., 2014). We included two measurements, identification and gist. The identification measure counted a description as correct if it included enough detail for an independent person to identify the scene. The gist measure calculated the percentage of gist items recollected for each image. The gist items were defined as any element pertaining to the scene's story that could not be changed or excluded without changing the main theme. Each image contained 2 to 4 predetermined gist items by (Küpper et al., 2014). The identification measure thus reflected whether participants could recollect the overall scene, and the gist measure reflected their ability to recall meaningful details of the scene.

\section{Experiment 2 \\ Participants}

The sample size was determined in advance via power analysis on the overall amnesic shadow effect in Experiment 1 (power $=85 \%,=0.05$, see https://osf.io/m3bhv for the preregistration). We recruited a sample of 48 adults (aged 18 to 29,39 females) with normal or corrected-to-normal vision. We excluded no participants based on the results of the consciousness check test. We obtained informed consent in accordance with procedures approved by the Human Subject Review Committee of the Shaanxi Normal University.

\section{Materials}

Experiment 2 used the materials from Experiment 1. We used three subsets of critical bystander items throughout the three experimental phases. As in Experiment 1, each bystander item was composed of an object-scene pair and a word-scene pair, encoded in separate learning phases. Implementing the consciousness check task also required a further set of foils composed of object cues from unstudied bystander pairs. Thus, in total, for each participant, across the bystander exposure task, the bystander final test, and the bystander consciousness check tasks, we used 18 double-cue/one-target bystander items, and 6 
bystander object cues. We also used the same 48 word ${ }_{5}$ pairs for the TNT task as we used in Experiment 1.

\section{Procedure}

Experiment 2 used the same three-phase procedure as Experiment 1. One key procedural change, however, was that in the TNT phase, participants reported their ongoing consciousness state on a trial-by-trial basis during the subliminal presentation period (Figure 4). Specifically, during the subliminal presentation period, the bystander object cue appeared in a sequence of 6 repetitions of F-MS-M-M-S-M as in Experiment 1. To implement the online consciousness checking task, during this 6-s period, participants made an immediate key press whenever they could consciously perceive the cue object. Whenever participants pressed a key in this manner, they then verbally reported the content of any perceived image. Finally, at the end of each full trial, participants further made an old/new judgement on the preceding masked bystander cue, regardless of whether the stimulus was reported to be consciously perceived. This old/new judgement was self-paced.

Implementing this new online consciousness checking task required that we introduce a new condition to the experiment. Specifically, to accommodate the need to make an old/new episodic discrimination at the end of each trial, we needed unstudied foil objects, for this discrimination to make sense. To achieve this, we added a new set of No-Think bystander trials during which an entirely novel never-before-studied bystander object cue was embedded between two No-Think trials (hereinafter, the No-Think-unconscious-novel condition). For this purpose, we designed a fourth subset of 6 bystander pairs that participants didn't study. We used the cue objects from these pairs as the bystander object during No-ThinkUnconscious-novel trials. Rather than increasing the number of trials during the TNT phase, we simply eliminated the No-Think-Conscious condition of Experiment 1 (which was not needed in Experiment 2) and replaced them with No-Think-Unconscious novel trials. Because we added No-Think-unconscious novel condition, for clarity we refer to the No-Think-unconscious condition of Experiment 1 as No-Think-unconscious-old condition, to distinguish them from No-Think-unconscious-novel trials. The testing phase adopted the same procedure as in Experiment 1. No offline consciousness check was given at the end of the experiment.

\section{Data analysis}

The analysis of the consciousness check task employed a different procedure than Experiment 1. First, because we forced participants to verbally report the content of any object cues that they claimed to see, our new procedure greatly cut down on participants' tendency to randomly guess during their detection decision. This enabled us to simply eliminate items that were subjectively reported to be visible at the very beginning of data analysis. Critically, because a trial-by-trial consciousness check proce- dure was used, items may appear conscious in some trials and unconscious in other trials. To avoid any influence of conscious awareness, any items that had been reported as consciously perceivable even just once were excluded from analyses. After eliminating the consciously recognized items, the recognition accuracy for all participants was calculated. No participant exceeded a recognition accuracy of $68.1 \%$ for the remaining bystander cues and therefore we retained all participants. Scoring of recall performance for the TNT and bystander recall tasks was the same as that used in Experiment 1.

\section{References}

Anderson, M. C., \& Green, C. (2001). Suppressing unwanted memories by executive control. Nature, 410(6826), 366-369. https : / / doi .org / 10 . 1038/ 35066572

Anderson, M. C., \& Hulbert, J. C. (2021). Active forgetting: Adaptation of memory by prefrontal control. Annual Review of Psychology, 72(1), annurev-psych-072720 094140. https://doi .org/10.1146/annurev-psych072720-094140

Anderson, M. C., Ochsner, K. N., Kuhl, B. A., Cooper, J., Robertson, E., Gabrieli, S. W., Glover, G. H., \& Gabrieli, J. D. E. (2004). Neural systems underlying the suppression of unwanted memories. Science, 303(5655), 232235. https://doi.org/10.1126/science.1089504

Benoit, R. G., \& Anderson, M. C. (2012). Opposing mechanisms support the voluntary forgetting of unwanted memories. Neuron, 76(2), 450-460. https://doi.org/10. 1016/j.neuron.2012.07.025

Benoit, R. G., Davies, D. J., \& Anderson, M. C. (2016). Reducing future fears by suppressing the brain mechanisms underlying episodic simulation. Proceedings of the National Academy of Sciences, 113(52), E8492-E8501. https://doi.org/10.1073/pnas.1606604114

Bradley, R., Greene, J., Russ, E., Dutra, L., \& Westen, D. (2005). A multidimensional meta-analysis of psychotherapy for PTSD. Am J Psychiatry, 162, 214-227.

Brewin, C. R., Gregory, J. D., Lipton, M., \& Burgess, N. (2010). Intrusive images in psychological disorders: Characteristics, neural mechanisms, and treatment implications. Psychological Review, 117(1), 210-232. https: //doi.org/10.1037/a0018113

Cleeremans, A. (2011). The radical plasticity thesis: How the brain learns to be conscious. Frontiers in Psychology, 2. https://doi.org/10.3389/fpsyg.2011.00086

Degonda, N., Mondadori, C. R., Bosshardt, S., Schmidt, C. F., Boesiger, P., Nitsch, R. M., Hock, C., \& Henke, K. (2005). Implicit associative learning engages the hippocampus and interacts with explicit associative learning. Neuron, 46(3), 505-520. https://doi.org/10.1016/ j.neuron.2005.02.030

Duss, S. B., Reber, T. P., Hänggi, J., Schwab, S., Wiest, R., Müri, R. M., Brugger, P., Gutbrod, K., \& Henke, K. (2014). Unconscious relational encoding depends on hippocampus. Brain, 137(12), 3355-3370. https://doi. org/10.1093/brain/awu270 
Henke, K. (2010). A model for memory systems based on processing modes rather than consciousness. Nature 60 Reviews Neuroscience, 11(7), 523-532. https://doi.org/ 61 10.1038/nrn2850

Henke, K., Mondadori, C. R., Treyer, V., Nitsch, R. M., 6 Buck, A., \& Hock, C. (2003). Nonconscious formation and reactivation of semantic associations by way of the 65 medial temporal lobe. Neuropsychologia, 41(8), 863- 66 876. https://doi.org/10.1016/S0028-3932(03)00035- 67 6

Hulbert, J. C., \& Anderson, M. C. (2018). What doesn't kill you makes you stronger: Psychological trauma and its relationship to enhanced memory control. Journal of Experimental Psychology: General, 147(12), 1931-1949. https://doi.org/10.1037/xge0000461

Hulbert, J. C., Henson, R. N., \& Anderson, M. C. (2016). Inducing amnesia through systemic suppression. Nature Communications, 7(1), 11003. https:// doi.org/10. 1038/ncomms11003

Jensen, K., Kirsch, I., Odmalm, S., Kaptchuk, T. J., \& Ingvar, M. (2015). Classical conditioning of analgesic and hyperalgesic pain responses without conscious awareness. Proceedings of the National Academy of Sciences, 112(25), 7863-7867. https://doi.org/10.1073/pnas. 1504567112

Kennedy, J., \& Taddonio, J. (1976). Experimenter effects in parapsychological research. The Journal of Parapsychology, 40, 1-33.

Küpper, C. S., Benoit, R. G., Dalgleish, T., \& Anderson, M. C. (2014). Direct suppression as a mechanism for controlling unpleasant memories in daily life. Journal of Experimental Psychology: General, 143(4), 1443-1449. https://doi.org/10.1037/a0036518

Loerinc, A. G., Meuret, A. E., Twohig, M. P., Rosenfield, D., Bluett, E. J., \& Craske, M. G. (2015). Response rates for CBT for anxiety disorders: Need for standardized criteria. Clinical Psychology Review, 42, 72-82. https: //doi.org/10.1016/j.cpr.2015.08.004

Pernet, C. R., Wilcox, R., \& Rousselet, G. A. (2013). Robust correlation analyses: False positive and power validation using a new open source matlab toolbox. Frontiers in Psychology, 3. https://doi.org/10.3389/fpsyg.2012. 00606

Taschereau-Dumouchel, V., Cortese, A., Chiba, T., Knotts, J. D., Kawato, M., \& Lau, H. (2018). Towards an unconscious neural reinforcement intervention for common fears. Proceedings of the National Academy of Sciences, 115(13), 3470-3475. https://doi.org/10.1073/pnas. 1721572115

Wang, Y., Luppi, A., Fawcett, J., \& Anderson, M. C. (2019). Reconsidering unconscious persistence: Suppressing unwanted memories reduces their indirect expression in later thoughts. Cognition, 187, 78-94. https://doi.org/ 10.1016/j.cognition.2019.02.016

Xu, X., Li, J., \& Chen, H. (2021). Valence and arousal ratings for 11,310 simplified chinese words. Behavior Research Methods. https://doi.org/10.3758/s13428021-01607-4
Zayfert, C., DeViva, J. C., Becker, C. B., Pike, J. L., Gillock, K. L., \& Hayes, S. A. (2005). Exposure utilization and completion of cognitive behavioral therapy for PTSD in a "real world" clinical practice. Journal of Traumatic Stress, 18(6), 637-645. https://doi.org/10.1002/jts.20072

Zhu, Z., \& Wang, Y. (2021). Forgetting unrelated episodic memories through suppression-induced amnesia. Journal of Experimental Psychology: General, 150(3), 401413. https://doi.org/10.1037/xge0000782 